\title{
Approach to the Workup and Management of Headache in the Emergency Department and Inpatient Settings
}

\author{
Morris Levin, $\mathrm{MD}^{1}$ \\ ${ }^{1}$ Division of Headache Medicine, Department of Neurology, University \\ of California, San Francisco, California \\ Address for correspondence Morris Levin, MD, Headache Center, \\ UCSF Department of Neurology, 2330 Post Street, San Francisco, \\ CA 94115 (e-mail: Morris.levin@ucsf.edu). \\ Semin Neurol 2015;35:667-674.
}

\begin{abstract}
Keywords

- headache

- migraine

- emergency

- inpatient

The challenge of headache management in the hospital can be divided into the diagnostic and management issues encountered in the emergency department, and the issues involved in the inpatient management of chronic refractory headaches. The two endeavors are different but linked because of overlapping diagnostic and treatment options. In addition, the problem of the intractable acute headache encountered in the emergency department can evolve into inpatient care of the same patient who has failed to respond to acute treatment. Here the author presents an approach to the patient with severe acute headache in the emergency department and explores the options available for managing intractable headaches in the inpatient setting.
\end{abstract}

The acute severe headache always warrants attention and often leads patients to the emergency department (ED). Here, there are several good pharmacologic options, as well as several pitfalls to be avoided, both diagnostically and therapeutically. A separate, but at times overlapping, problem is the patient with intractable chronic headache, an agonizingly painful and frustrating situation for patients, families, and medical team members. Often, these patients will have tried many approaches with knowledgeable physicians to no avail, and inpatient evaluation and treatment can be useful in selected cases. In this review an approach to the emergency or urgent care patient presenting with severe headache is outlined, beginning with diagnostic considerations, followed by a selection of options for management. Then the inpatient management of the "refractory headache" patient is addressed, beginning with suggested principles for selecting patients who might benefit from hospitalization, followed by a description of inpatient treatment choices and methods.

\section{Headache in the Emergency Department}

Headache is extremely common in the emergency department (ED)-accounting for more than 5 million ED visits in the United States in 2011. ${ }^{1}$ The major diagnostic pitfalls in approaching the patient with severe acute headache in the ED are misdiagnosis of primary headache syndromes and undiagnosed secondary causes of headache. Missing the correct primary headache diagnosis will reduce the chance of successful treatment. Missing a secondary headache cause can have disastrous consequences. Fortunately, only approximately $5 \%$ of patients with severe headache seen the ED will have secondary headaches. ${ }^{2}$ Red flags such as meningismus, fever, neurologic signs, and concurrent medical illnesses should raise concern ( - Table $\mathbf{1}$ ).

In taking a history in acute headache patients (-Table 2 ), mode of onset is very important, with a rapid evolution to severe pain suggesting secondary causes. Exertion during or before onset is also worrisome, although it can be a benign presentation. Severity is important, but most patients in the ED will probably endorse a high level of pain intensity. Asking the patient if it is the worst headache they have ever had is not particularly useful. Focal neurologic symptoms suggest neurologic disease, such as arterial dissection, intracranial mass or vasculitis. Current medications (such as sedatives, stimulants, and anticoagulants) and other substances must be known so potential causes of headache as well as potential
Issue Theme Hospitalist Neurology; Guest Editors: S. Andrew Josephson, MD, and Vanja C. Douglas, MD
Copyright $\odot 2015$ by Thieme Medical Publishers, Inc., 333 Seventh Avenue, New York, NY 10001, USA. Tel: +1(212) 584-4662.
DOI http://dx.doi.org/ 10.1055/s-0035-1564300. ISSN 0271-8235. 
Table 1 Red flags in patients with headache

\begin{tabular}{|l|}
\hline New (recent onset or change in pattern) \\
\hline Effort-induced or positional \\
\hline Late life onset (middle age or later) \\
\hline Meningismus, fever \\
\hline AIDS, cancer, or other known systemic illness \\
\hline Neurologic or psychological symptoms or signs \\
\hline
\end{tabular}

Table 2 History-taking in acute headache

\begin{tabular}{|l|}
\hline Mode of onset (fast vs. slow) \\
\hline Exertion during onset or before \\
\hline Location \\
\hline Focal symptoms \\
\hline Current medications and drugs \\
\hline Family history \\
\hline Medical illnesses \\
\hline
\end{tabular}

roadblocks to treatment can be clarified. The presence of concurrent medical illnesses, such as diabetes, HIV infection and neoplastic disease, must be understood for similar reasons.

Examining the patient with acute headache (- Table 3 ) must include an assessment of meningismus as a clue to meningeal inflammation due to infection or hemorrhage. Eye examination, including careful pupillary evaluation, is very helpful to exclude ocular disease, such as acute angle-closure glaucoma. Funduscopic evaluation is essential as an indicator of intracranial pressure. Head and neck evaluation should include palpation of the paranasal sinuses, temporomandibular joint regions, submandibular tissues, carotids, temporal arteries, and supraorbital regions. A complete neurologic exam is essential. The Dix-Hallpike maneuver may be helpful when vertigo is a key accompaniment. Provocative maneu-

Table 3 Examination criteria in acute headache

\begin{tabular}{|l|}
\hline Vital signs \\
\hline Meningismus \\
\hline $\begin{array}{l}\text { Eye exam including pupillary light response } \\
\text { and funduscopy }\end{array}$ \\
\hline $\begin{array}{l}\text { Head and neck exam - sinuses, temporomandibular } \\
\text { region, submandibular areas, } \\
\text { carotid arteries, superficial nerves }\end{array}$ \\
\hline Neurologic exam \\
\hline Dix-Hallpike maneuver \\
\hline
\end{tabular}

vers may help clarify cervical spine disease, including the Spurling maneuver.

Routine laboratory testing in acute headache may be of low yield, but serum glucose, electrolytes, blood cell counts, and pregnancy testing are generally recommended. Computed tomography (CT) is essential for patients who have had recent head trauma or in whom a mass lesion or subarachnoid hemorrhage is suspected. Computed tomography may be overused in evaluating acute headache, as approximately 95\% demonstrate no abnormality. ${ }^{2}$ The American College of Emergency Physicians asserted in a policy statement there was Level B ("moderate clinical certainty") evidence for neuroimaging in acute headache patients with (1) new-onset severe headache, (2) new abnormal neurologic findings, or (3) HIV infection. ${ }^{3}$ Computed tomography angiography (CTA) can be very helpful when any of the following is suspected: aneurysmal rupture or expansion, reversible cerebral vasoconstriction syndrome, cerebral vasculitis, cerebral venous thrombosis (with CT venography [CTV]), or arterial dissection (carotid or vertebral). ${ }^{4}$ Magnetic resonance imaging (MRI) is rarely indicated in acute headache in the absence of focal neurologic symptoms suggestive of stroke or intracranial mass. Lumbar puncture (LP) is mandatory if meningitis or subarachnoid hemorrhage is suspected, as would be the case in a patient with meningismus, fever, or a history of very rapidly evolving head pain.

By far the most common cause of severe acute headache in the ED is migraine, ${ }^{2,5}$ although it is difficult to know exactly how common because migraine is underdiagnosed in ED settings. ${ }^{6}$ The diagnosis of migraine is often patently clear to ED clinicians when the patient has a history of severe migrainous headaches requiring acute treatment. But when the patient's symptoms are not entirely explained by migraine or if there are red flags, further workup for secondary causes is indicated (-Table $\mathbf{4}$ ).

A worrisome presentation is the thunderclap headache, which occurs either instantaneously or very close to it. Spontaneous thunderclap headache suggests subarachnoid hemorrhage and the best approach is CT of the head followed by LP because it has been estimated that a small fraction (as high as 7\%) of normal CT exams are false-negatives ${ }^{7}$ (see - Table 5 for causes of thunderclap headache).

Cerebral venous thrombosis can be difficult to diagnose; standard MRI sequences may fail to identify even large thrombi in cerebral veins. Magnetic resonance or CT venography, however, is almost always diagnostic. The syndrome of reversible cerebral vasoconstriction syndrome (RCVS), also known as Call-Fleming syndrome, often presents as sudden or severe headache and only later manifests neurologic deficits. Unlike central nervous system vasculitis, cerebrospinal fluid in RCVS is generally normal. Fortunately, CTA seems to be almost as useful as standard conventional angiography in demonstrating the hallmark segmental arterial narrowing. It is important to remember that RCVS may lead to focal subarachnoid bleeding, which can lead to a fruitless quest for a ruptured aneurysm.

Carotid or vertebral arterial dissection can present with acute severe headache and without other neurologic 
Table 4 Secondary causes of acute headache

\begin{tabular}{|l|}
\hline Intracranial hemorrhage (subarachnoid, parenchymal) \\
\hline Cerebral venous thrombosis \\
\hline Reversible cerebral vasoconstriction syndrome (RCVS) \\
\hline Carotid or vertebral artery dissection \\
\hline Acute posttraumatic (postconcussive) headache \\
\hline Hydrocephalus \\
\hline Meningitis \\
\hline Encephalitis \\
\hline Acute angle closure glaucoma \\
\hline Acute sinusitis \\
\hline Systemic infection \\
\hline Medication-induced headache \\
\hline Intracranial mass \\
\hline
\end{tabular}

symptoms or antecedent neck trauma, and can be very challenging to detect without vascular imaging. Sphenoid sinusitis may also present as sudden and or severe nonlocalizing head pain, but should be seen easily on MRI or sinus CT. Spontaneous intracranial hypotension, generally diagnosed by the patient's complaint of worsening of pain upon sitting or standing and improvement with reclining, may present with severe and/or thunderclap headache as well.

There are several primary headache disorders other than migraine that may present to the ED. Cluster headache is usually easily diagnosed by its unilaterality, accompanying cranial autonomic signs (tearing, flushing, nasal congestion, and discharge), and episodic nature with each headache

Table 5 Causes of sudden or rapidly evolving (thunderclap) headache

\begin{tabular}{|l|}
\hline $\begin{array}{l}\text { Subarachnoid hemorrhage or sentinel } \\
\text { headache (“aneurysmal leak") }\end{array}$ \\
\hline Hypertensive intracranial hemorrhage \\
\hline Pituitary apoplexy \\
\hline Cerebral venous thrombosis \\
\hline Carotid or vertebral artery dissection \\
\hline Spontaneous intracranial hypotension \\
\hline Cerebral vasculitis \\
\hline Reversible cerebral vasoconstriction syndrome (RCVS) \\
\hline Acute hypertension \\
\hline Primary thunderclap headache \\
\hline Sex-related headache \\
\hline Exertional headache \\
\hline Migraine \\
\hline
\end{tabular}

lasting less than 2 hours. Hemicrania continua is an incessant, sometimes severe, side-locked headache that will only respond to indomethacin. Exertional and sex-related headaches may have thunderclap onset, but these both tend to be selflimited and are largely treatable with nonsteroidal antiinflammatory medications (NSAIDs). Finally, there is the "primary thunderclap headache," which is benign and idiopathic; like most primary headaches, it is a diagnosis of exclusion.

When secondary headaches and the rare forms of primary headache have been excluded, management of severe migraine becomes the focus. Most patients will have already tried oral analgesics like aspirin, acetaminophen, and NSAIDs. Many will have significant nausea. As a result, most patients will respond best to parenteral analgesics. There is limited evidence for most pharmacologic options used to treat acute migraine in the ED. - Table 6 summarizes the choices with best evidence, or at least the greatest acceptance by authorities in the field. I will discuss each and attempt to provide a useful paradigm for treating severe migraine.

\section{Neuroleptic Antiemetics}

Chlorpromazine 25 to $50 \mathrm{mg}$ intravenously (IV), prochlorperazine $10 \mathrm{mg}$ IV, and metoclopramide $10 \mathrm{mg}$ IV are all useful options. There is very good evidence supporting the effectiveness of all of these. ${ }^{8-11}$ The analgesic mechanism of these medications in migraine is unknown. Adverse effects include dystonia, which is generally preventable by concurrent treatment with diphenhydramine $25 \mathrm{mg}$. Akathisia is another unpleasant side effect, which may also be preventable with diphenhydramine. Orthostatic hypotension, sedation, and cardiac conduction changes (particularly QT interval prolongation) may also occur.

\section{Triptans and Ergots}

Similar to other ergot family members, dihydroergotamine (DHE) has a mild vasoconstrictive effect that affects primarily veins rather than arteries. Adverse effects are mainly confined to mild nausea, muscle cramps, mild blood pressure

Table 6 Options for acute treatment of migraine in the emergency department setting

\begin{tabular}{|l|}
\hline Ketorolac 30-60 mg IM \\
\hline Chlorpromazine $25-50 \mathrm{mg}$ IV \\
\hline Prochlorperazine $10 \mathrm{mg}$ IV \\
\hline Metoclopramide $10 \mathrm{mg}$ IV \\
\hline Dihydroergotamine $1 \mathrm{mg}$ IV, IM \\
\hline Sumatriptan $6 \mathrm{mg} \mathrm{SC}$ \\
\hline Valproate $250-500 \mathrm{mg}$ IV \\
\hline Magnesium $1-4 \mathrm{~g}$ IV \\
\hline Dexamethasone $4-10 \mathrm{mg}$ IV \\
\hline Morphine $5-10 \mathrm{mg} \mathrm{IV}$ \\
\hline
\end{tabular}

Abbreviations: IM, intramuscular; IV, intravenous; SC, subcutaneous. 
elevation, and sedation. It is helpful to concurrently administer an antiemetic, which helps migraine-related nausea as well as DHE-induced nausea. Dihydroergotamine is available for intramuscular (IM) and IV use; a dose of 0.5 to $1 \mathrm{mg}$ is effective in adults. It is contraindicated in patients with cardiac disease or significant hypertension.

Sumatriptan is an excellent choice for migraine treatment in the ED. The subcutaneous injection of $6 \mathrm{mg}$ is dramatically effective in many patients, and side effects are generally minimal, consisting of transient paresthesias, muscle tightness, dizziness, or sedation. Dihydroergotamine and triptan medications are surprisingly underused in the ED setting despite their known significant benefits as migraine abortives. $^{12}$ This may be due to unwarranted concern about adverse effects or simply lack of familiarity with them.

\section{Parenteral Nonsteroidal Anti-Inflammatory Agents}

Intramuscular ketorolac is a common choice for acute migraine treatment in the ED, supported by good evidence. ${ }^{13-15}$ It can be given IV at a dose of $30 \mathrm{mg}$, or IM generally at a dose of $60 \mathrm{mg}$. It has a benign adverse effect profile, although nephrotoxicity is a concern when given over time. Other adverse effects include gastrointestinal disturbance, weakness, and dizziness. Diclofenac sodium IM has also been reported as useful in acute migraine.

\section{Valproate}

Divalproex sodium can be used in a dose of 500 to $1000 \mathrm{mg}$ diluted in $50 \mathrm{ml}$ of normal saline and infused over 30 minutes. Possible adverse effects of dizziness and somnolence are generally transient. Efficacy has not been well proven, despite several attempts at treatment trials. One study compared IV valproate with ketorolac and metoclopramide; it was the least effective of the three. ${ }^{11}$

\section{Magnesium}

Some patients with acute severe migraine will respond to magnesium sulfate $1 \mathrm{~g}$ IV administered over 10 to 30 minutes. This may be repeated up to a total of $5 \mathrm{~g}$. Fair evidence exists for this intervention, including a study by Demirkaya et al in 2001, in which 13 of 15 patients became pain free after treatment. ${ }^{12,16,17}$ Caution should be used in patients with renal insufficiency.

\section{Opioids}

Opioids are the most commonly prescribed agents for acute migraine in the acute setting ${ }^{18}$ despite their relative ineffectiveness in pain relief ${ }^{19}$ and clear association with increased rates of return to the ED. ${ }^{20}$ They do not seem to have a beneficial effect on terminating migraine attacks, but can temporarily suppress some pain on the basis of their effects on mu opioid receptors. Adverse effects from doses high enough to reduce head pain include constipation, urinary retention, nausea, edema, and pruritus, and in susceptible patients, seizures and respiratory depression. But the primary reason to avoid them is their tendency over time in patients with chronic pain conditions to lead to tolerance, dependence and addiction, all of which can happen insidiously, beginning in the ED. When other options are not possible, morphine 5 to $10 \mathrm{mg}$ IM, 2 to $5 \mathrm{mg}$ IV, or hydromorphone 2 to $4 \mathrm{mg}$ IV are potential choices.

\section{Corticosteroids}

The goal of acute migraine treatment must include prevention of recurrence as well as pain relief. Here corticosteroids seem to be of help and have become part of a routine approach in some centers. ${ }^{21}$ A common choice is a single dose of dexamethasone 4 to $10 \mathrm{mg}$ IV. Because repeated doses of steroids can lead to aseptic bone necrosis, it is important to know if there is recurrent use of steroids in headache patients being seen in the ED.

\section{Peripheral Nerve Blockade}

Greater occipital nerve blockade has become more popular in the acute relief of severe migraine pain. ${ }^{22}$ Evidence is lacking because it has been challenging to design a controlled study where the sham treatment is not easily identifiable by subjects. Adverse effects in skilled hands are minimal, and relief can be rapid. Local anesthetics injected in the scalp will be absorbed systemically; side effects may include lightheadedness. A common choice of local anesthetic agents is a 1:1 mixture of $1 \%$ lidocaine and $0.25 \%$ bupivacaine injected into the greater occipital nerve trunk region. Although it is common practice to add steroid medication to these injections, it has no proven benefit. ${ }^{23}$

\section{Antinauseants}

Relieving nausea can be accomplished with the neuroleptic antinauseants in doses discussed above. In addition to these, ondansetron, a serotonin-3 receptor antagonist, can be very useful as an IV injection of 4 to $8 \mathrm{mg}$ slowly pushed. Hydroxyzine, an antihistamine, is also effective parenterally, at a dose of 25 to $50 \mathrm{mg}$ IV or IM. Hydration is essential when the patient is or has been vomiting, so IV access must be established early if this is the case.

\section{Children}

Like adults, many children who go to the ED with headache have migraine or other primary headaches, but secondary headaches, especially those due to infection, are more common. Hence, a very careful evaluation is needed. Although pediatric treatment of migraine in the ED follows the above approaches, there is very little evidence for the use of these medications in children. The benefits of neuroleptic medications and ketorolac are supported by reasonably good evidence. $^{24-26}$ Dihydroergotamine doses in the range of $0.25 \mathrm{mg}$ IV for preteens and 0.5 to $1.0 \mathrm{mg}$ IV for teens have been effective. Subcutaneous sumatriptan seems to be very effective in adolescents and children, but has not been rigorously tested. Hydration is particularly important in children who have been vomiting.

\section{Pregnancy}

Treatment choices for severe migraine in pregnancy are limited. Magnesium intravenously has been helpful for 
many patients, as has occipital nerve blockade. Hydration alone can sometimes be of help.

Guidelines for ED treatment of acute migraine have been difficult to craft, in part because of disparate ways of assessing success in different studies and also because of interpatient variation. The Canadian Association of Emergency Physicians published guidelines that resemble practice in many academic EDs, with dopamine antagonists (neuroleptic antinauseants), serotonin receptor antagonists (DHE, triptans), NSAIDs, and corticosteroids being most highly recommended. ${ }^{27}$ A paradigm for the assessment and management of acute headache in the ED is shown in - Fig. 1.

Sadly, results of acute headache treatment in the ED are not impressive. One study found that only $22 \%$ of patients were pain free on discharge from a university ED, and that $64 \%$ of patients discharged with improvement had a return of severe headache within 24 hours. ${ }^{6}$ Part of any ED approach to treating acute migraine (or any acute episode of a chronic headache condition) is to lay the groundwork for future evaluation and prophylactic treatment by neurologists or other headache specialists. This can be arranged at the time of discharge or soon thereafter by ED staff, knowing that if this is not done they may likely see the patient again in the near future. Unfortunately, the same study noted that only $40 \%$ of patients were asked to follow up with a physician. ${ }^{6}$

\section{Inpatient Treatment of Refractory Headaches}

Most chronic headache disorders can be managed on an outpatient basis with prophylactic and "rescue" (analgesic/ abortive) medication along with careful management of lifestyle triggers. But some patients fail to respond. It is difficult to know how many patients would benefit from inpatient treatment, but given the fact that approximately $4 \%$ of the adult population have daily headaches, ${ }^{28}$ the need probably dwarfs present capacity.
Table 7 International Classification of Headache Disorders criteria for medication overuse headache

Headache occurring on $\geq 15$ days per month in a patient with a preexisting headache disorder

Regular overuse for $>3$ months of one or more drugs that can be taken for acute and/or symptomatic treatment of headache

Not better accounted for by another ICHD-III diagnosis

Abbreviations: ICDH, International Classification of Headache Disorders. Source: Reprinted with permission from the International Headache Society.

Most patients with refractory headache meet the International Headache Society criteria for chronic migraine, have medication-overuse headache $(\mathrm{MOH})$ (-Table 7), or both. There are several other causes of refractory headache, including refractory cluster headache, refractory hemicrania continua, posttraumatic headaches, high or low intracranial pressure headaches, neuralgias, and other missed secondary headaches. However, with modern diagnostic technology most of the secondary causes have been excluded long before the patient is seen in a center offering inpatient headache treatment.

Medication overuse headache poses special problems. Although $\mathrm{MOH}$ pathophysiology is still unclear, it seems that many migraine patients who use large amounts of analgesic medications will not respond to prophylactic approaches until the use of the analgesic medications dramatically lessens. Specific quantities of rescue medication required for the production of $\mathrm{MOH}$, and best ways to reverse it, are being studied. Clearly, for some patients, careful reduction and discontinuation of previous analgesics is required for a successful outcome. This should ideally start at home prior to admission, with further work in the hospital. Interestingly, patients with $\mathrm{MOH}$ often have the best results following inpatient headache treatment. ${ }^{29}$

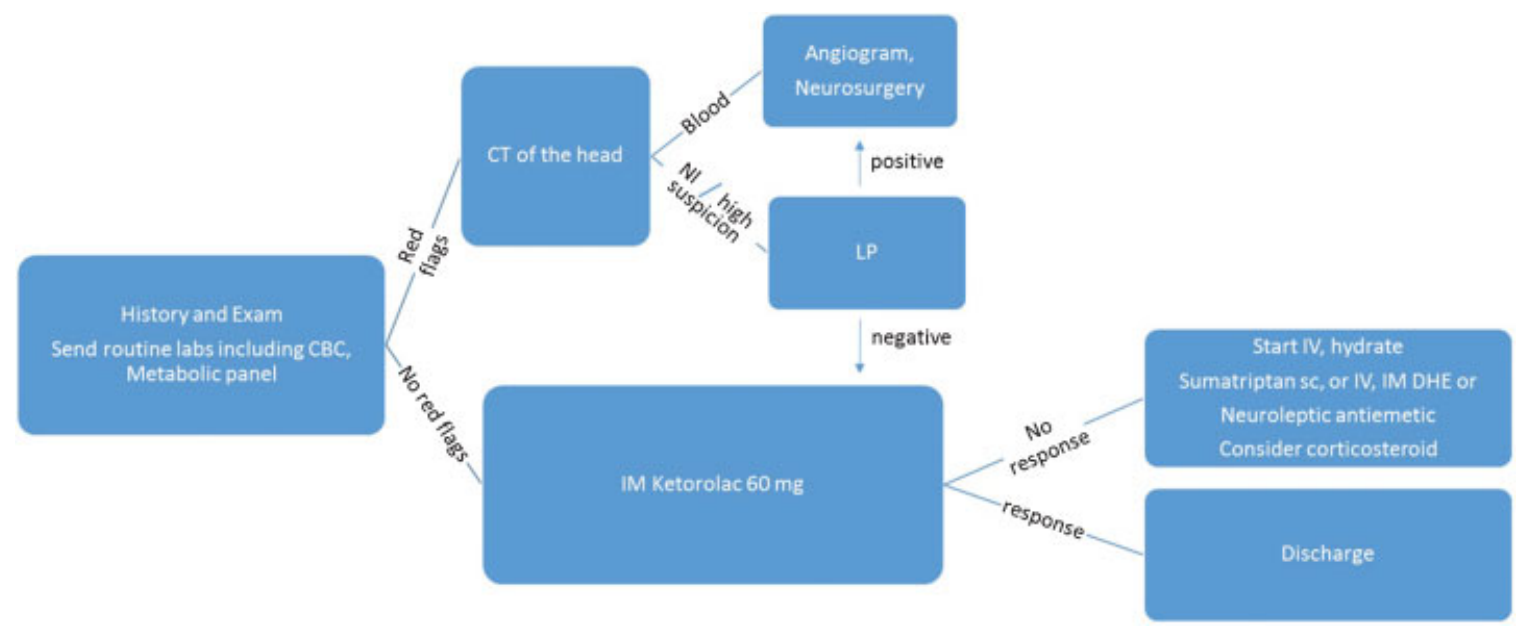

Fig. 1 Schematic for the treatment of adults with acute headache in the emergency department. CBC, complete blood count; CT, computed tomography; DHE, dihydroergotamine; IM, intramuscular; IV, intravenous; LP, lumbar puncture; NI, normal; SC, subcutaneous. 
Which refractory migraine patients should be admitted for inpatient treatment? Clearly, patients who are experiencing daily or near daily headaches and who have been unresponsive to several prophylactic and acute interventions are good candidates. These patients must be carefully evaluated for overlooked primary or secondary headaches, which would have prevented response to migraine-specific treatment. Previous treatment must be scrutinized carefully to make sure that "failed" treatments were delivered in appropriate doses and over enough time to see results. Comorbid conditions need to be elucidated to make sure confounding issues, such as chronic medical or psychiatric illnesses, have not been preventing response to migraine treatment. Other indications for inpatient treatment might include the need to withdraw safely from analgesic medications and confounding medical or psychiatric conditions that require close supervision during medication trials. Finally, in some instances where acute pain or nausea seem to be progressing to dangerous levels, admission for rehydration and parenteral analgesia is a sensible course.

What are the goals of inpatient chronic migraine treatment? Although the primary goal may be to reduce the frequency and severity of headaches and associated symptoms like nausea, the inpatient program should also aim for (and evaluate the outcomes of) other goals such as decreasing disability, reducing the use of rescue medications, reducing the numbers of office and ED visits, and improving quality of life. What makes the process of inpatient treatment challenging is that most or all of these factors may be unknowable until some time following discharge from the hospital. This should be made clear to patients so that expectations are not falsely elevated.

Opportunities for management of refractory migraine on an inpatient basis can be divided into several categories: (1) IV courses of medication, (2) withdrawal from medications thought to be causing medication-overuse headache, (3) biobehavioral and other nonpharmacologic treatment such as lifestyle coaching, (4) adjustment of prophylactic medications, (5) trials of new acute treatments, (6) diagnostic testing for possible missed conditions, and (7) anesthetic procedures. It is beyond the scope of this article to address all of these, other than to encourage their exploration. I will address IV pharmacologic protocols that have been helpful for some patients, and address several other key issues including the treatment of medication overuse.

The literature in the area of IV management programs for refractory headache is largely observational with a paucity of controlled studies. The IV agent that has the best evidence for reversing intractable migraine is DHE, which was first proposed by Raskin and has been used widely. ${ }^{30}$ Adverse effects are manageable, and results are generally good for many patients. Intravenous chlorpromazine has likewise been used widely with good results, although controlled trials are lacking. Intravenous lidocaine, dexamethasone, divalproex, ketamine, and propofol have also been used in selected cases of refractory headache conditions. Most of these approaches can produce results rapidly, but more often improvement is noted after discharge. Pitfalls in inpatient IV headache treat- ment are common and include psychiatric and medical illness flares during treatment or following discharge, difficult to manage pain exacerbations during treatment, insufficient patient education, and poor follow-up postdischarge.

\section{Dihydroergotamine}

Raskin reported a series of 55 patients with long-term constant headache prior to admission, most of whom responded dramatically to 0.3 to $1 \mathrm{mg}$ DHE every 8 hours for 2 days. ${ }^{30}$ Silberstein reported similarly good results in a mixed series including some patients with the notoriously resistant "new daily persistent headache." ${ }^{31}$ Authors of that study also found persistent significant improvement 2 years after admission. Several variations on Raskin's original protocol have been proposed. Best results may occur with 4- to 5day infusions. ${ }^{32}$ Dihydroergotamine's mechanism of action is not fully understood, but it is known to bind brainstem serotonergic centers like the dorsal raphe nucleus, which are implicated in migraine etiopathology. Why IV courses of DHE might produce lasting effects is also not known, but its prolonged effect might be related to its long-lived active metabolite, 8-hydroxy-DHE.

The dose of IV DHE to establish efficacy seems to need to be in the 0.5 - to 1 -mg range given 3 times daily. Adverse effects include nausea and vomiting, muscle cramping, joint aches, diarrhea, chest discomfort, and paresthesias. The nausea can be limiting, but is generally well controlled with metoclopramide or other antinauseants. A common strategy is to pretreat prior to each DHE dose with 10-mg IV metoclopramide, often after diphenhydramine $25 \mathrm{mg}$ IV. Toxic effects of vasospasm, tachycardia, bradycardia, hypertension, and ergotism are quite uncommon with the typical doses used in refractory migraine, but contraindications to the use of DHE are peripheral vascular disease, cardiovascular or cerebrovascular disease, uncontrolled hypertension, liver or renal failure, and pregnancy.

\section{Chlorpromazine}

Although there is no compelling evidence for the efficacy of IV chlorpromazine in the inpatient treatment of intractable migraine, it has gained popularity in several headache centers. Adverse effects include sedation, dystonia, akathisia, orthostatic hypotension, prolonged QT interval, and rarely, priapism. Contraindications include previously intolerable and unpreventable dystonia or akathisia, baseline prolonged QT interval, and pregnancy. Blood pressure should be monitored. Electrocardiography should be done daily to monitor the corrected QT interval (QTc), and each dose should be pretreated with diphenhydramine $25 \mathrm{mg}$ orally or IV. Keeping patients in bed unless aided carefully is best to avoid falls.

A typical initial dose is $12.5 \mathrm{mg}$ IV every 6 to 8 hours. It seems that best results occur when the patient is maintained in at least a lightly sedated state. If this does not happen relatively soon in the course of treatment, dosage can be adjusted upwards by $12.5 \mathrm{mg}$ at each step, with a maximum of approximately $50 \mathrm{mg} 3$ times daily. The regimen is typically continued for 3 to 4 days. 


\section{Corticosteroids}

Another anecdotally helpful regimen is IV corticosteroid medication for several days. Dexamethasone IV 2 mg every 8 hours for 2 to 3 days has been effective in selected patients. It has also been used in combination with IV DHE and is said to help in reducing withdrawal symptoms related to the reduction or discontinuation of previous analgesics. Another option is methylprednisolone 100 to $200 \mathrm{mg}$ IV every 12 hours for 3 days. Aseptic necrosis of the hip or other bones is unlikely with a short course, but with repetitive courses of parenteral or oral steroids the risk rises. Other potential adverse effects are fluid retention, hypertension, hyperglycemia, nausea, insomnia, irritability, and hypertension.

\section{Valproate}

Repeated doses of $500 \mathrm{mg}$ diluted in $20-\mathrm{ml}$ normal saline infused over several minutes every 8 hours over 3 days can be helpful. This regimen was as effective as dihydroergotamine and metoclopramide in one small study. ${ }^{33}$ Another approach is to administer $1000 \mathrm{mg}$ once daily for 3 days. Possible adverse effects include dizziness and somnolence.

\section{Propofol}

This IV sedative-hypnotic has been proposed for intractable migraine. ${ }^{34}$ It induces a hypnotic state rapidly at nonanesthetic doses in the range of 20- to $30-\mathrm{mg}$ IV push, which is repeated every 5 minutes until light sedation is induced. Close monitoring of respirations and airway protection is essential. Another report corroborated its usefulness, noting resolution of headache in 6 out of 18 patients and improvement in 11 patients. $^{35}$

\section{Lidocaine}

Lidocaine infused at 2 to $4 \mathrm{mg} / \mathrm{min}$ in D5W (5\% dextrose in water) has been reported to help in two retrospective reviews. ${ }^{36,37}$ Treatment is started at a low rate of infusion at 1 $\mathrm{mg} / \mathrm{min}$ with continuous cardiac telemetry and blood pressure monitoring aiming to keep systolic blood pressure between 90 to $160 \mathrm{~mm} \mathrm{Hg}$, diastolic between 45 to $95 \mathrm{~mm}$ $\mathrm{Hg}$, and pulse 50 to $120 \mathrm{bpm}$. Side effects include nausea, hypotension, arrhythmias, and occasional hallucinations, most of which are generally manageable. The optimal duration of infusion is not clear, but seems to be longer than for DHE or neuroleptics, perhaps on the order of 6 to 14 days.

\section{Inpatient Treatment of Medication Withdrawal and Other Symptomatic Management}

For patients with medication-overuse headache, most headache specialists will make rigorous efforts to discontinue analgesic medications. Opioid withdrawal symptoms can be alleviated to some extent with clonidine, $0.1 \mathrm{mg}$ every 8 hours as needed, while carefully monitoring blood pressure. Barbiturate withdrawal can be mitigated with benzodiazepines, such as lorazepam, with an attempt to quickly wean off to prevent benzodiazepine dependence.

Nausea associated with treatment or withdrawal can be managed with neuroleptic antinauseants, ondansetron, or hydroxyzine. Pain flares should not be treated with opioids, but may respond to NSAIDs such as oral naproxen sodium or IM ketorolac. Psychiatric conditions may be triggered by the change in environment and/or by pain, and should be managed when possible with nonpharmacologic approaches including relaxation techniques and psychotherapy if available. Insomnia can be treated with melatonin; hydroxyzine or diphenhydramine may also be of help.

\section{Inpatient Treatment of Children}

Intravenous DHE with metoclopramide seems quite helpful for children with chronic intractable migraine. Linder reported the results of this program in 30 children aged 6 to 12 years: 24 had either a 90\% improvement in headache frequency, headache intensity, or both. Dose was adjusted for age between 0.1 to $0.5 \mathrm{mg}$ per dose. ${ }^{38}$ Combining IV DHE and prochlorperazine has met with success. ${ }^{39}$ The importance of psychological assessment and counseling in children with intractable headache must be emphasized; when this is added to medical treatment, children seem to have far better long-term outcomes. ${ }^{40}$

\section{Posthospital Planning}

Discharge planning is essential and must start before admission. Realistic expectations should be established, including the understanding that improvement in pain might only be apparent following hospitalization. Clear prophylactic and acute medication instructions must be conveyed. A firm follow-up outpatient appointment should be made within several weeks of discharge. Nonpharmacologic strategies, such as relaxation techniques, exercise, meal and sleep regulation, and avoidance of sedatives and other substances should be coached. Good psychological support when indicated should be assured, and support systems (family and friends) should be identified and discussed.

\section{Conclusions}

Most headache patients who are evaluated in EDs have migraine, but there are some other primary and secondary headache disorders that must be ruled out depending upon the details of presentation. Once this is done, several options for relief of head pain are available, including parenteral ketorolac, neuroleptic antiemetics, DHE, triptans, and magnesium sulfate. Opioids, while commonly employed, are generally less useful, and may lead to readmission to the ED.

Although most chronic headache disorders can be managed satisfactorily in the outpatient setting, inpatient treatment offers several advantages, particularly in patients who are overusing medications. Intravenous DHE has good evidence for efficacy. Other IV regimens using neuroleptic antiemetics, lidocaine, valproate, and corticosteroids have all been effective in many reported cases.

Few commonly proposed treatments for acute headache relief in the ED have been studied systematically in that setting; similarly, most inpatient IV treatment protocols are not clearly proven to be effective. Obstacles to further research include lack of funding and individual differences between refractory headache patients, making comparison studies difficult. 


\section{References}

1 Lucado J, Paez K, Elixhauser A. Headaches in U.S. Hospitals and Emergency Departments, 2008. HCUP Statistical Brief \#111. Rockville, MD: Agency for Healthcare Research and Quality; 2011

2 Goldstein JN, Camargo CA Jr, Pelletier AJ, Edlow JA. Headache in United States emergency departments: demographics, work-up and frequency of pathological diagnoses. Cephalalgia 2006;26(6): 684-690

3 Edlow JA, Panagos PD, Godwin SA, Thomas TL, Decker WW; American College of Emergency Physicians. Clinical policy: critical issues in the evaluation and management of adult patients presenting to the emergency department with acute headache. Ann Emerg Med 2008;52(4):407-436

4 Alons IME, van den Wijngaard IR, Verheul RJ, et al. The value of CT angiography in patients with acute severe headache. Acta Neurol Scand 2015;131(3):164-168

5 Sahai-Srivastava S, Desai P, Zheng L. Analysis of headache management in a busy emergency room in the United States. Headache 2008;48(6):931-938

6 Gupta MX, Silberstein SD, Young WB, Hopkins M, Lopez BL, Samsa GP. Less is not more: underutilization of headache medications in a university hospital emergency department. Headache 2007; 47(8):1125-1133

7 Byyny RL, Mower WR, Shum N, Gabayan GZ, Fang S, Baraff LJ. Sensitivity of noncontrast cranial computed tomography for the emergency department diagnosis of subarachnoid hemorrhage. Ann Emerg Med 2008;51(6):697-703

8 Cameron JD, Lane PL, Speechley M. Intravenous chlorpromazine vs intravenous metoclopramide in acute migraine headache. Acad Emerg Med 1995;2(7):597-602

9 Kostic MA, Gutierrez FJ, Rieg TS, Moore TS, Gendron RT. A prospective, randomized trial of intravenous prochlorperazine versus subcutaneous sumatriptan in acute migraine therapy in the emergency department. Ann Emerg Med 2010;56(1):1-6

10 Kelley NE, Tepper DE. Rescue therapy for acute migraine, part 2: neuroleptics, antihistamines, and others. Headache 2012;52(2): 292-306

11 Friedman BW, Garber L, Yoon A, et al. Randomized trial of IV valproate vs metoclopramide vs ketorolac for acute migraine. Neurology 2014;82(11):976-983

12 Kelley NE, Tepper DE. Rescue therapy for acute migraine, part 1 : triptans, dihydroergotamine, and magnesium. Headache 2012; 52(1):114-128

13 Seim MB, March JA, Dunn KA. Intravenous ketorolac vs intravenous prochlorperazine for the treatment of migraine headaches. Acad Emerg Med 1998;5(6):573-576

14 Shrestha M, Singh R, Moreden J, Hayes JE. Ketorolac vs chlorpromazine in the treatment of acute migraine without aura. A prospective, randomized, double-blind trial. Arch Intern Med 1996; 156(15):1725-1728

15 Taggart E, Doran S, Kokotillo A, Campbell S, Villa-Roel C, Rowe BH. Ketorolac in the treatment of acute migraine: a systematic review. Headache 2013;53(2):277-287

16 Gertsch E, Loharuka S, Wolter-Warmerdam K, Tong S, Kempe A, Kedia S. Intravenous magnesium as acute treatment for headaches: a pediatric case series. J Emerg Med 2014;46(2): 308-312

17 Demirkaya S, Vural O, Dora B, Topçuoğlu MA. Efficacy of intravenous magnesium sulfate in the treatment of acute migraine attacks. Headache 2001;41:171-177

18 Kelley NE, Tepper DE. Rescue therapy for acute migraine, part 3: opioids, NSAIDs, steroids, and post-discharge medications. Headache 2012;52(3):467-482

19 Tepper SJ. Opioids should not be used in migraine. Headache 2012; 52(Suppl 1):30-34

20 Tornabene SV, Deutsch R, Davis DP, Chan TC, Vilke GM. Evaluating the use and timing of opioids for the treatment of migraine headaches in the emergency department. J Emerg Med 2009; 36(4):333-337

21 Colman I, Friedman BW, Brown MD, et al. Parenteral dexamethasone for acute severe migraine headache: meta-analysis of randomised controlled trials for preventing recurrence. BMJ 2008; 336(7657):1359-1361

22 Ashkenazi A, Blumenfeld A, Napchan U, et al; Interventional Procedures Special Interest Section of the American. Peripheral nerve blocks and trigger point injections in headache management - a systematic review and suggestions for future research. Headache 2010;50(6):943-952

23 Ashkenazi A, Matro R, Shaw JW, Abbas MA, Silberstein SD. Greater occipital nerve block using local anaesthetics alone or with triamcinolone for transformed migraine: a randomised comparative study. J Neurol Neurosurg Psychiatry 2008;79(4):415-417

24 Brousseau DC, Duffy SJ, Anderson AC, Linakis JG. Treatment of pediatric migraine headaches: a randomized, double-blind trial of prochlorperazine versus ketorolac. Ann Emerg Med 2004;43(2): 256-262

25 Leung S, Bulloch B, Young C, Yonker M, Hostetler M. Effectiveness of standardized combination therapy for migraine treatment in the pediatric emergency department. Headache 2013;53(3): 491-197

26 Bachur RG, Monuteaux MC, Neuman MI. A comparison of acute treatment regimens for migraine in the emergency department. Pediatrics 2015;135(2):232-238

27 Ducharme J. Canadian Association of Emergency Physicians guidelines for the acute management of migraine headache. J Emerg Med 1999;17(1):137-144

28 Stewart WF, Lipton RB, Celentano DD, Reed ML. Prevalence of migraine headache in the United States. Relation to age, income, race, and other sociodemographic factors. JAMA 1992;267(1): 64-69

29 Diamond S, Freitag FG, Maliszewski M. Inpatient treatment of headache: long-term results. Headache 1986;26(4):189-197

30 Raskin NH. Repetitive intravenous dihydroergotamine as therapy for intractable migraine. Neurology 1986;36(7):995-997

31 Silberstein SD, Silberstein JR. Chronic daily headache: long-term prognosis following inpatient treatment with repetitive IV DHE. Headache 1992;32(9):439-445

32 Nagy AJ, Gandhi S, Bhola R, Goadsby PJ. Intravenous dihydroergotamine for inpatient management of refractory primary headaches. Neurology 2011;77(20):1827-1832

33 Mathew NT, Kailasam J. Repetitive intravenous administration of valproate sodium in intractable migraine: comparison with intravenous dihydroergotamine (DHE). Neurology 2000;54:A22 (abstract)

34 Krusz JC, Scott V, Belanger J. Intravenous propofol: unique effectiveness in treating intractable migraine. Headache 2000;40(3): 224-230

35 Mendes PM, Silberstein SD, Young WB, Rozen TD, Paolone MF Intravenous propofol in the treatment of refractory headache. Headache 2002;42(7):638-641

36 Williams DR, Stark RJ. Intravenous lignocaine (lidocaine) infusion for the treatment of chronic daily headache with substantial medication overuse. Cephalalgia 2003;23(10):963-971

37 Rosen N, Marmura M, Abbas M, Silberstein S. Intravenous lidocaine in the treatment of refractory headache: a retrospective case series. Headache 2009;49(2):286-291

38 Linder SL. Treatment of acute childhood migraine headaches. Cephalalgia 1991;11(Suppl 11):120-121

39 Kabbouche MA, Powers SW, Segers A, et al. Inpatient treatment of status migraine with dihydroergotamine in children and adolescents. Headache 2009;49(1):106-109

40 Lanzi G, D'Arrigo S, Termine C, et al. The effectiveness of hospitalization in the treatment of paediatric idiopathic headache patients. Psychopathology 2007;40(1):1-7 